\title{
Development, implementation, and prospective validation of a model to predict 60-day end-of-life in hospitalized adults upon admission at three sites
}

Vincent J. Major *iD and Yindalon Aphinyanaphongs

\begin{abstract}
Background: Automated systems that use machine learning to estimate a patient's risk of death are being developed to influence care. There remains sparse transparent reporting of model generalizability in different subpopulations especially for implemented systems.

Methods: A prognostic study included adult admissions at a multi-site, academic medical center between 2015 and 2017. A predictive model for all-cause mortality (including initiation of hospice care) within 60 days of admission was developed. Model generalizability is assessed in temporal validation in the context of potential demographic bias. A subsequent prospective cohort study was conducted at the same sites between October 2018 and June 2019. Model performance during prospective validation was quantified with areas under the receiver operating characteristic and precision recall curves stratified by site. Prospective results include timeliness, positive predictive value, and the number of actionable predictions.

Results: Three years of development data included 128,941 inpatient admissions (94,733 unique patients) across sites where patients are mostly white (61\%) and female (60\%) and $4.2 \%$ led to death within 60 days. A random forest model incorporating 9614 predictors produced areas under the receiver operating characteristic and precision recall curves of $87.2(95 \% \mathrm{Cl}, 86.1-88.2)$ and $28.0(95 \% \mathrm{Cl}, 25.0-31.0)$ in temporal validation. Performance marginally diverges within sites as the patient mix shifts from development to validation (patients of one site increases from 10 to 38\%). Applied prospectively for nine months, 41,728 predictions were generated in real-time (median [IQR], $1.3[0.9,32]$ minutes). An operating criterion of 75\% positive predictive value identified 104 predictions at very high risk (0.25\%) where 65\% (50 from 77 well-timed predictions) led to death within 60 days.

Conclusion: Temporal validation demonstrates good model discrimination for 60-day mortality. Slight performance variations are observed across demographic subpopulations. The model was implemented prospectively and successfully produced meaningful estimates of risk within minutes of admission.
\end{abstract}

Keywords: Mortality prediction, Palliative care, Supportive care, End-of-life care, Advance directives, Medical informatics, Machine learning, Electronic health records

\footnotetext{
* Correspondence: vincent.major@nyulangone.org

Department of Population Health, NYU Langone Health, 227 East 30th St, 6th Floor, New York, NY 10016, USA
} 


\section{Background}

\section{Supportive and palliative care}

Supportive care describes a myriad of interventions intended to prevent or improve symptoms of disease or side-effects of treatment. Patients with terminal illness often receive supportive care as their disease progresses towards palliative and end-of-life care. Unfortunately, many patients do not receive palliative care until their last weeks of life [1] despite guidelines recommending palliative care for any patient diagnosed with a chronic or serious illness that will ultimately lead to their death [2]. Practical methods to identify patients who would benefit from palliative and, more generally, supportive care and end-of-life planning are needed [3].

Physicians make treatment decisions-including whether to initiate or defer palliative care-based upon their perception of a patient's condition. Unfortunately, physicians tend to be optimistic when estimating prognosis [4-6]. Since the entire process relies on human judgement, patients who have previously been overlooked for supportive care can continue to slip through the cracks. Automated systems can augment clinician gestalt as a failsafe mechanism to improve quality and consistency of care.

\section{Mortality prediction}

Many systems have been developed to estimate mortality risk. Early methods developed scores to be applied, by hand, at the bedside with a small number of parameters. Promising recent works apply machine learning to predict mortality risk upon admission, or shortly afterwards, to prompt palliative care [7-9]. These works rely on high-level administrative data [9], claims data [10], billing codes from the electronic health record (EHR) [7, 11], or concepts extracted from clinical notes [8]. Many of these works focus on long-term mortality, typically 1year, or restrict to specific cohorts or datasets that limit their utility to influence care decisions. Although many models have been developed, few machine learning systems are implemented in clinical practice [12]. Even fewer studies have assessed model safety and performance across sites.

\section{Objective}

To develop and validate a machine learning model to predict short-term mortality at the instant of inpatient admission using EHR data. Model validation consists of two steps: first, model generalization is investigated by assessing testing set performance across sites. Second, the model is implemented and prospectively validated to assess technical feasibility and real-world performance before release into the EHR.

\section{Methods \\ Data \\ Study setting}

This prognostic study was conducted at NYU Langone Health, a multi-site academic medical center in New York City. At the time of model development, July 2018, NYU Langone Health consisted of approximately 1300 beds across one general and one Orthopedics hospital in the borough of Manhattan and one general hospital in Brooklyn.

This project met the definition of quality improvement outlined by the NYU Grossman School of Medicine IRB and is not considered human subjects research and did not require IRB approval. This study follows the reporting guidelines set out in the Transparent Reporting of a Multivariable Prediction Model for Individual Prognosis or Diagnosis (TRIPOD) statement.

\section{Patient population}

A retrospective dataset was selected by identifying all adults hospitalized between January 1, 2015 and December 31, 2017 (> 18 years old at admission). Admissions for inpatient hospice care were excluded along with observation stays but patients 'boarding' in the emergency department were included. No other inclusion criteria were imposed.

\section{Mortality outcomes}

All-cause death outcomes in the community can be problematic for predictive modeling as patients can die anywhere and reporting of deaths can vary widely. One of the primary challenges is missingness that can be caused by a variety of practical, social and technical reasons. Researchers often improve their data by combining several sources of data into a composite [13]. We follow this trend and exploit three available sources: 1) internal system-wide death data, 2) purchased death data (derived from the Social Security Administration's Master Death File), and 3) hospice discharge disposition data (both inpatient and home hospice). None of these mechanisms perfectly capture all deaths but, together, establish a measure of 'end-of-life' where the addition of hospice improves robustness but adds noise (eResults and eFigure 1).

After extensive discussion with physicians, a primary outcome of mortality within 60 days of admission was selected. The rationale for 60 days is to promote urgency in end-of-life decision-making while allowing sufficient time to initiate supportive and palliative care interventions, both during the hospitalization and in the community post-discharge. At the time of model development (July 2018), more than 6 months had passed since the end of the study period ensuring adequate time for 60day outcomes to accrue. 


\section{Feature construction}

To estimate risk within minutes of admission, all predictors must be reliably accessible at that time (i.e. data collected after arrival likely cannot be used). Instead, one year of historical data (up to the day prior) is considered for each admission. Patient demographics and discrete data describing prior encounters are collected along with several categories of coded data used in related works [7, 10, 11], namely: ICD-10 (International Classification of Diseases) diagnosis codes, CPT (Current Procedural Terminology) procedure codes, RxNorm medication codes, and LOINC (Logical Observation Identifiers Names and Codes) laboratory result codes. Each data type, except demographics, are dated in a patient's history and can occur many times.

Features are constructed from these data similarly to related works $[7,10,11]$. Specifically, each patient's history is segmented into four time slices with boundaries at 30, 90, and 180 days preceding admission [7], excluding all data collected more than a year prior. Each data category (e.g. ICD-10 diagnoses) is aggregated in each slice into:

- Count of each unique code,

- Count of unique codes and total code count across days, and

- Mean, variance, minimum, maximum, and range of the daily number of codes.

While a typical patient may have fewer than a dozen unique ICD-10 codes, all patients have aggregate values, e.g. total codes during slice and max number of daily codes. With these features, a model may learn differences in specific disease types as well as disease burden and utilization with these features.

\section{Experimental design}

A retrospective cohort-study experimental design that 'enrolls' each admission is employed with a temporally separated testing set. This design has been demonstrated in prior work [14] to improve implementation performance without overestimation during validation. Three years of data were partitioned into training and testing cohorts on January 1, 2017 resulting in 24 and 12 months respectively as described in Fig. 1a. No other cohort selection criteria were applied during the training period-all (re)admissions were included. Patients who were readmitted within the testing period (2017) following their 'enrollment' during the training period (2015/ 2016) are excluded to ensure no individual patient is present in both groups.

A dramatic increase in monthly admissions is evident in Fig. 1 as the NYU Langone Brooklyn hospital transitioned onto the existing EHR system in August 2016. The training cohort therefore underrepresents Brooklyn patients with only 4.5 months of data included (19\% from 24 months). This non-random underrepresentation during model derivation is a blatant example of a wider challenge faced by any predictive model: generalizing into a future population.

\section{Model development}

Many thousands of candidate predictors are expected where only a small fraction exist for a typical patient.
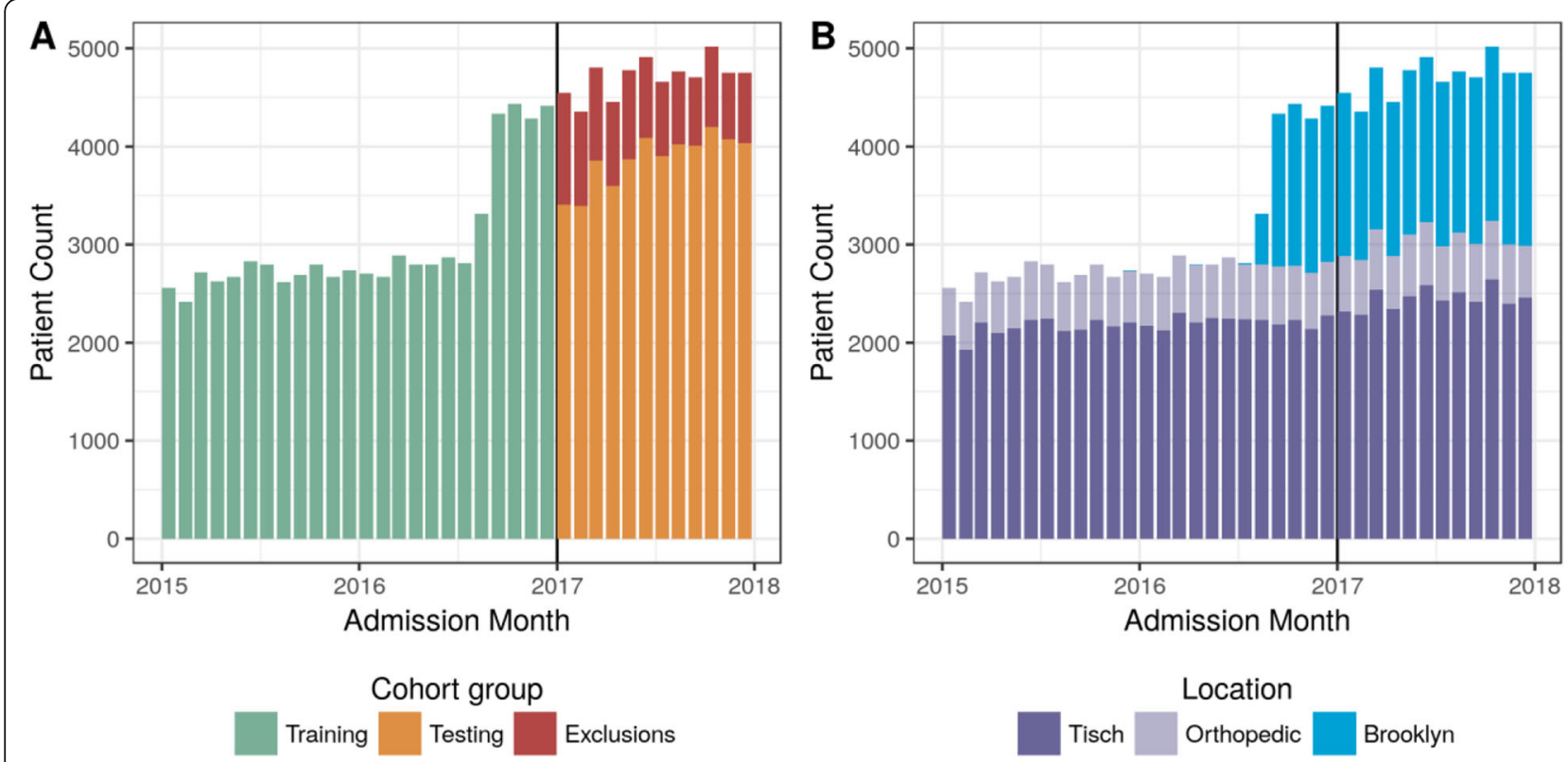

Fig. 1 Monthly admissions stratified by a) model development cohort and b) hospital location 
With this many predictors, overfitting to spurious associations and small samples is difficult to avoid. Predictors are pruned by requiring at least once occurrence in both outcome groups (i.e. survival and death) and a total count exceeding 100 . This leaves 9614 features (11\% of $87,226)$ for modeling. Some training cohort patients (19.6\%) are left with only demographic features and are removed from model training. No data imputation is performed.

Many of these features are sparse (e.g. specific ICD-10 codes), others are complete but highly nonlinear (e.g. count of procedure codes) which complicate modeling. An algorithm is needed that can learn which featuresand which values within those features-are prognostic. Three classifiers were considered (eMethods): logistic regression with lasso regularization [15], XGBoost [16], and random forest [17]. The later two are tree-based algorithms, known for their consistent performance on a variety of datasets [18] and within similar mortality work $[10,19]$. Parameter selection was conducted within 5fold cross validation by sampling patients (not admissions [20]) before retraining one model on the entire training set (eMethods). Model performance within the testing set is assessed with area under the receiver operating characteristic (AUROC) and area under the precision recall curve (AUPRC). To evaluate the impact of Brooklyn underrepresentation during training, testing set performance is compared across locations by considering principles of model transparency [21] and model fairness [22] (eMethods).

\section{Implementation}

\section{Operating threshold}

Before implementation, an operating threshold for intervention must be selected (eMethods). Clinical stakeholders selected a criterion of $75 \%$ positive predictive value (PPV), corresponding to one false positive from four high-risk predictions. The 75\% PPV criterion was imposed on the testing set under bootstrap conditions to calculate a robust threshold (eMethods).

\section{Prospective validation}

In order to assess the model's utility to influence care decisions, it was implemented and prospectively applied to patient data in a controlled manner. A 'silent-live' phase allowed prospective data to be collected but predictions were withheld from clinicians until initial results were assessed and any technical issues resolved. This interim period provides time to develop a clinical workflow.

After a successful silent-live period, the model was deployed live where it would deliver estimates of risk in near real-time to enable prospective validation. Data spanning nine months between October 2018 and June
2019 was used for analysis. During this period, all new inpatient admissions were collected but predictions were never made for children, nor those with no prior data (i.e. only demographics). For this prospective cohort study, patient data was collected to enable analysis of prediction volume, timeliness, and PPV when implemented live. Patient outcomes were only collected for patients identified at high-risk.

\section{Evaluation in the context of potential demographic Bias} Model fairness is an increasingly important factor affecting deployment of predictive models, especially in applications involving vulnerable populations. Model fairness is closely related to how a model generalizes across populations-particularly demographic groups-and impacts patient safety. Several recent works have reported that the explicit removal of 'sensitive' data elements-such as gender or race-may perpetuate inequalities observed within the data [22, 23]. In the interest of transparency [21] (one pillar of responsible machine learning), model performance in different strata of sensitive demographics is investigated (eMethods, eResults, eFigures 5 and 6). One model, trained on the entire training set is applied to sub-cohorts of the testing cohort by combinations of sensitive demographics (e.g. Black women admitted in Brooklyn) and various measures of model performance are reported. This procedure is repeated for a second model where all sensitive demographics are excluded or 'masked' during training (eMethods and eResults).

\section{Results}

Patient cohort and outcomes

In the three calendar years considered, 128,941 inpatient admissions occurred across the three hospitals including 94,733 unique patients. The population is mostly white (61\%) and female (60\%). Patient demographics with location, comorbidity, and outcome characteristics are reported in Table 1. The underrepresentation of Brooklyn patients in the training cohort and considerable structural differences between locations (eTable 1) lead to differences when comparing training and testing sets. Of all admissions, $4.2 \%$ led to death or hospice within 60 days and the median time from admission to outcome is 53 days with no drastic differences by cohort or demographics (eFigure 2).

\section{Retrospective modeling \\ Performance within the training cohort}

Sampling from the training set produced five comparable folds for cross-validation, each with a similar number of patients $(14,230-14,617)$ and outcome rate (3.9-4.3\%). AUROC and AUPRC within cross-validation from each model is reported in Table 2. The random forest classifier with 100 trees and a maximum depth of 
Table 1 Demographics, outcome, comorbidity, and model predictor characteristics of the model development population

\begin{tabular}{|c|c|c|c|c|}
\hline & $\begin{array}{l}\text { All Patients } \\
n=128,941\end{array}$ & $\begin{array}{l}\text { Training Set } \\
n=72,437\end{array}$ & $\begin{array}{l}\text { Testing Set } \\
n=46,458\end{array}$ & \\
\hline \multicolumn{5}{|l|}{ Demographics $^{a}$} \\
\hline Measure & & & & \\
\hline Age & $\%(n)$ & $\%(n)$ & $\%(n)$ & * \\
\hline $18-29$ & $11.5 \%(14786)$ & $10.7 \%(7778)$ & $13.1 \%(6087)$ & \\
\hline $30-39$ & $17.5 \%(22607)$ & $18.0 \%(13053)$ & $18.0 \%(8361)$ & \\
\hline $40-49$ & $9.45 \%(12183)$ & $9.49 \%(6877)$ & 9.69\% (4504) & \\
\hline $50-59$ & $13.3 \%(17204)$ & $13.5 \%(9784)$ & $13.4 \%(6206)$ & \\
\hline $60-69$ & $18.2 \%(23500)$ & $18.7 \%(13556)$ & $17.3 \%(8026)$ & \\
\hline 70-79 & $15.8 \%(20388)$ & $15.8 \%(11439)$ & $15.1 \%(7008)$ & \\
\hline $80-89$ & $10.7 \%$ (13839) & $10.5 \%(7588)$ & $10.2 \%(4748)$ & \\
\hline $90+$ & $3.44 \%$ (4434) & $3.26 \%(2362)$ & $3.27 \%(1518)$ & \\
\hline Ethnicity ${ }^{b}$ & $\%(n)$ & $\%(n)$ & $\%(n)$ & * \\
\hline Hispanic & $9.75 \%(3467)$ & $9.77 \%(2336)$ & $8.62 \%(666)$ & \\
\hline Not Hispanic & $90.3 \%$ (32086) & $90.2 \%(21584)$ & $91.4 \%(7060)$ & \\
\hline Unknown & -- (93388) & -- (48517) & -- (38732) & \\
\hline Race & $\%(n)$ & $\%(n)$ & $\%(n)$ & * \\
\hline Black & $10.9 \%(14033)$ & $11.0 \%(7933)$ & $10.7 \%(4987)$ & \\
\hline East Asian & $7.38 \%(9520)$ & $6.50 \%(4707)$ & $9.10 \%(4230)$ & \\
\hline West Asian & $1.66 \%(2146)$ & $1.68 \%(1219)$ & $1.74 \%(807)$ & \\
\hline White & $61.6 \%(79424)$ & $64.1 \%(46404)$ & $57.3 \%(26642)$ & \\
\hline Other & $16.4 \%(21181)$ & $14.8 \%(10692)$ & $18.8 \%(8714)$ & \\
\hline Unknown & $2.05 \%(2637)$ & $2.05 \%(1482)$ & $2.32 \%(1078)$ & \\
\hline Sex & $\%(n)$ & $\%(n)$ & $\%(n)$ & \\
\hline Female & $60.1 \%(77478)$ & $60.3 \%(43664)$ & $60.5 \%(28130)$ & \\
\hline Male & $39.9 \%$ (51459) & $39.7 \%$ (28770) & $39.4 \%$ (18327) & \\
\hline Unknown & $0 \%(4)$ & $0 \%(3)$ & $0 \%(1)$ & \\
\hline Site & $\%(n)$ & $\%(n)$ & $\%(n)$ & * \\
\hline Tisch & $63.4 \%(81807)$ & $72.3 \%$ (52398) & $49.2 \%(22877)$ & \\
\hline Orthopedic & $15.6 \%$ (20137) & $18.1 \%(13122)$ & $12.8 \%(5938)$ & \\
\hline Brooklyn & $20.9 \%$ (26997) & $9.55 \%(6917)$ & $38 \%(17643)$ & \\
\hline Outcomes $^{c}$ & $\%(n)$ & $\%(n)$ & $\%(n)$ & \\
\hline Any known death & 7.93\% (10229) & $9.00 \%(6521)$ & $5.20 \%(2414)$ & * \\
\hline \multirow[t]{2}{*}{ 60-day death } & $4.15 \%(5356)$ & $4.05 \%(2935)$ & $3.57 \%(1657)$ & * \\
\hline & Median [IQR] & Median [IQR] & Median [IQR] & \\
\hline Days from admission to death & $53[6,205]$ & $83[12,306]$ & $21[1,92.75]$ & * \\
\hline Comorbidities $^{d}$ & Median [IQR] & Median [IQR] & Median [IQR] & \\
\hline \multirow[t]{2}{*}{ Charlson Score } & $1[0,2]$ & $1[0,2]$ & $0[0,2]$ & * \\
\hline & $\%(n)$ & $\%(n)$ & $\%(n)$ & \\
\hline AIDS/HIV & $0.626 \%(635)$ & $0.61 \%(349)$ & $0.506 \%(176)$ & \\
\hline Cancer (any malignancy) & $16.8 \%(17094)$ & $18.2 \%(10432)$ & $13.2 \%(4594)$ & * \\
\hline Cerebrovascular disease & $10.0 \%(10149)$ & $9.99 \%(5716)$ & $8.13 \%(2826)$ & * \\
\hline Chronic obstructive pulmonary disease & $17.9 \%(18218)$ & $18.6 \%(10649)$ & $13.5 \%(4703)$ & * \\
\hline Congestive heart failure & $12.0 \%(12144)$ & $11.8 \%$ (6774) & $8.56 \%$ (2978) & * \\
\hline
\end{tabular}


Table 1 Demographics, outcome, comorbidity, and model predictor characteristics of the model development population (Continued)

\begin{tabular}{|c|c|c|c|c|}
\hline & $\begin{array}{l}\text { All Patients } \\
n=128,941\end{array}$ & $\begin{array}{l}\text { Training Set } \\
n=72,437\end{array}$ & $\begin{array}{l}\text { Testing Set } \\
n=46,458\end{array}$ & \\
\hline Dementia & $3.67 \%(3721)$ & $3.18 \%(1819)$ & $3.09 \%(1075)$ & \\
\hline Diabetes with chronic complications & $6.34 \%(6439)$ & $4.9 \%(2806)$ & $5.68 \%(1977)$ & * \\
\hline Diabetes without chronic complications & $16.8 \%(17019)$ & $16.2 \%(9256)$ & $14.4 \%(4995)$ & * \\
\hline Hemiplegia or paraplegia & $2.92 \%(2962)$ & $2.83 \%(1617)$ & $2.35 \%(817)$ & * \\
\hline Metastatic solid tumor & $6.02 \%(6115)$ & $6.39 \%(3657)$ & $4.55 \%(1584)$ & * \\
\hline Mild liver disease & $6.40 \%(6495)$ & $6.23 \%(3566)$ & $5.14 \%(1787)$ & * \\
\hline Moderate or severe liver disease & $1.62 \%(1642)$ & $1.59 \%(910)$ & $1.11 \%(385)$ & * \\
\hline Myocardial infarction & $9.73 \%(9874)$ & $9.48 \%(5423)$ & $6.9 \%(2400)$ & * \\
\hline Peptic ulcer disease & $1.84 \%(1871)$ & $1.76 \%(1009)$ & $1.27 \%(443)$ & * \\
\hline Peripheral vascular disease & $13.1 \%(13278)$ & $13.0 \%(7446)$ & $9.97 \%(3469)$ & * \\
\hline Renal disease & $10.9 \%(11093)$ & $10.4 \%(5937)$ & $7.93 \%(2759)$ & * \\
\hline Rheumatoid disease & $2.87 \%(2915)$ & $3.11 \%(1781)$ & $2.06 \%(718)$ & * \\
\hline
\end{tabular}

\section{Predictors}

\begin{tabular}{|c|c|c|c|c|c|}
\hline Range & Measure & Median [IQR] & Median [IQR] & & \\
\hline $1-30$ days & \# of diagnoses & $3[0,12]$ & $3[0,13]$ & $2[0,10]$ & * \\
\hline $1-30$ days & \# of lab results & $0[0,46]$ & $3[0,47]$ & $0[0,43]$ & * \\
\hline $1-30$ days & \# of office visits & $3[1,6]$ & $3[1,6]$ & $2[1,5]$ & * \\
\hline $1-30$ days & \# of emergency department visits & $0[0,0]$ & $0[0,0]$ & $0[0,0]$ & * \\
\hline $1-30$ days & \# of hospitalizations & $0[0,0]$ & $0[0,0]$ & $0[0,0]$ & * \\
\hline 1-365 days & \# of diagnoses & $15[2,51]$ & $14[2,52]$ & $11[0,36]$ & * \\
\hline $1-365$ days & \# of lab results & $35[0,151]$ & $34[0,142]$ & $15[0,84]$ & * \\
\hline 1-365 days & \# of office visits & $11[5,25]$ & $11[5,25]$ & $9[4,20]$ & * \\
\hline $1-365$ days & \# of emergency department visits & $0[0,1]$ & $0[0,1]$ & $0[0,1]$ & \\
\hline $1-365$ days & \# of hospitalizations & $0[0,1]$ & $0[0,1]$ & $0[0,0]$ & * \\
\hline
\end{tabular}

*: Differences between training and testing sets are computed with: 1) $x^{2}$ tests for demographics; 2) proportion tests for individual comorbidities and mortality rates; and 3) Mann-Whitney tests for Charlson score and days from admission to death. In all cases, statistical significance is indicated $\left(^{*}\right)$ for adjusted $p<0.05$ using a Bonferroni correction

a: Demographics coded within the EHR at the time of admission

b: Ethnicity contains many missing values which are omitted before computing the proportion and difference between groups

c: Including death and initiation of hospice care

d: Comorbidities are derived from ICD-10 diagnosis codes present in each patient's year of history pre-admission using the diagnostic groups of the Charlson Comorbidity Index as implemented in the comorbidity R package [24]. Patients with no documented history are omitted from the denominator of each comorbidity

Table 2 Model performance within cross-validation, applied to the testing set, and stratified by site

\begin{tabular}{|c|c|c|c|c|}
\hline Model & Cohort & Measure & AUROC & AUPRC \\
\hline Lasso regression & $\begin{array}{l}\text { Training } \\
\text { (Cross-validation) }\end{array}$ & $\begin{array}{l}\text { Mean } \\
{[\text { min, max] }}\end{array}$ & $\begin{array}{l}78.8 \\
{[78.0,80.2]}\end{array}$ & $\begin{array}{l}21.0 \\
{[18.3,22.0]}\end{array}$ \\
\hline XGBoost & $\begin{array}{l}\text { Training } \\
\text { (Cross-validation) }\end{array}$ & $\begin{array}{l}\text { Mean } \\
{[\text { min, max }]}\end{array}$ & $\begin{array}{l}84.6 \\
{[83.8,86.0]}\end{array}$ & $\begin{array}{l}25.7 \\
{[21.2,27.4]}\end{array}$ \\
\hline \multirow[t]{4}{*}{ Random forest } & $\begin{array}{l}\text { Training } \\
\text { (Cross-validation) }\end{array}$ & $\begin{array}{l}\text { Mean } \\
{[\text { min, max }]}\end{array}$ & $\begin{array}{l}86.9 \\
{[85.3,87.7]}\end{array}$ & $\begin{array}{l}26.4 \\
{[20.1,31.0]}\end{array}$ \\
\hline & $\begin{array}{l}\text { Testing } \\
\text { (Bootstrapped) }\end{array}$ & $\begin{array}{l}\text { Median } \\
{[95 \% \mathrm{Cl}]}\end{array}$ & $\begin{array}{l}87.2 \\
{[86.1,88.2]}\end{array}$ & $\begin{array}{l}28.0 \\
{[25.0,31.0]}\end{array}$ \\
\hline & Brooklyn & $\begin{array}{l}\text { Median } \\
{[95 \% \mathrm{Cl}]}\end{array}$ & $\begin{array}{l}83.8 \\
{[81.9,85.6]}\end{array}$ & $\begin{array}{l}26.6 \\
{[22.5,31.0]}\end{array}$ \\
\hline & Non-Brooklyn & $\begin{array}{l}\text { Median } \\
{[95 \% \text { Cl] }}\end{array}$ & $\begin{array}{l}88.9 \\
{[87.5,90.2]}\end{array}$ & $\begin{array}{l}30.1 \\
{[26.4,33.7]}\end{array}$ \\
\hline
\end{tabular}


1000 (eResults) outperformed the lasso regression model with marginal improvement over the XGBoost model and was selected as the final model (Table 2). The most influential predictors are aggregates that describe utilization (eTable 2).

\section{Performance within the testing cohort}

When applied to the testing cohort, the random forest model performs similarly to cross-validation, with median and 95\% confidence intervals (CIs) for AUROC and AUPRC reported in Table 2. The receiver operating characteristics and precision-recall curves of the entire testing set are described in Fig. 2 with the selected threshold highlighted. (Calibration is assessed in eResults and eFigure 3). Each category of data contributes to overall redundancy where removing any one has little to no effect on overall performance (eTable 3).

\section{Performance across sites within the testing cohort}

Table 2 also describes model performance within the Brooklyn sub-cohort, reporting a marginal decrease in AUROC, compared to the Non-Brooklyn group (combining Manhattan hospitals as both are faithfully represented). This divergence is visible within Fig. 2a. A similar pattern is observed in AUPRC, as described in Table 2 and Fig. 2b. Despite the visible region of decreased performance towards the bottom-left corner, the three curves of Fig. 2b overlap at PPV above 50\% where our supportive care application is likely to operate. That is, the marginally worse global performance does not impact localized performance. (Calibration across locations is also assessed in eResults and eFigure 3).

\section{Operating threshold within the testing cohort}

A criterion for $75 \%$ PPV yields an operating threshold of 0.355 with $4.6 \%$ sensitivity/recall where patients exceeding this threshold are at very high risk of dying (eFigure $2 \mathrm{~B})$. Less than $5 \%$ of all deaths in the 60 days following admission can be predicted while maintaining this strict PPV constraint (no more than one false positive from four high-risk predictions). All-cause mortality includes entirely unpredictable deaths, increasing the denominator and shrinking sensitivity. This $4.6 \%$ group constitutes a real, potentially impactful group of patients at very high risk of dying.

Although the location-specific precision-recall curves of Fig. 2b overlap at 75\% PPV, the corresponding thresholds are not guaranteed to be similar. Distributions of predicted probabilities across the three hospitals suggests high similarity between the two general hospitals (Brooklyn and Tisch) except in the very high-risk range (eFigure 4). When the operating threshold is applied to only Brooklyn patients, PPV shifts up to $92 \%$ as highlighted in Fig. 2b. The distributional differences between Brooklyn and non-Brooklyn populations combined with the imbalanced training proportions result in
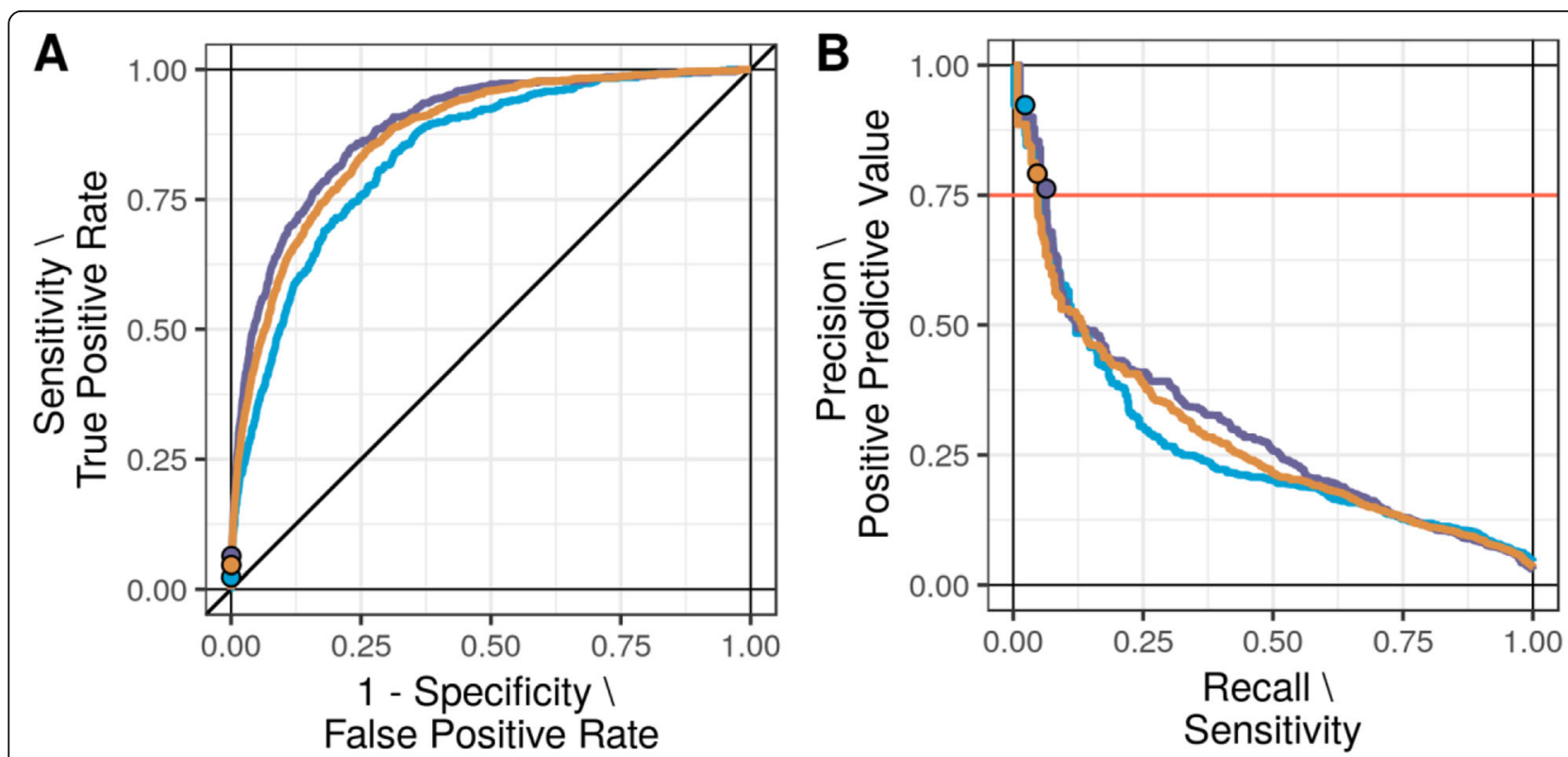

\section{Cohort}

$$
\text { - Testing - Brooklyn - Non-Brooklyn }
$$

Fig. 2 Performance curves from the complete testing cohort and further stratified by location. a receiver operating characteristic, and $\mathbf{b}$ precision-recall curves. The selected threshold is highlighted along with each corresponding point once stratified by location 
very precise, conservative application of the model to Brooklyn patients.

Application of one institution-wide threshold at Brooklyn poses little risk to patients but does perpetuate underrepresentation of Brooklyn patients. Several threshold-agnostic and threshold-specific measures of model performance are compared across locations and further across demographic sub-populations of sex, race, and ethnicity (eMethods, eResults, eFigures 5 and 6). Discrepancies in performance consistently underrepresent Brooklyn patients at very high risk of dying but similar differences are observed across other demographics. Removal of race and ethnicity as features worsens these disparities. This bias further highlights the need for transparency and pragmatic solutions to reach equity.

\section{Prospective validation}

Our model was silently tested for 12 weeks, beginning August 2018, by sending an once-a-day email to assess validity of patients above the operating threshold (and a sample below). The vast majority (78\%; 74 of 95$)$ of patients reviewed by a hospitalist were expected to benefit from supportive care. At the time of review, many of these patients were not, at least yet, receiving supportive care. Multiple comorbidities and complex disease were common, including patients who were not considered appropriate for intervention. During this period, a near real-time prediction system was developed to generate a prediction for each patient within minutes of admission.

After a successful silent-live period, the model was implemented live in October 2018. In the nine months through June 2019:

1. 49,785 inpatient admissions were detected for prediction,

2. 48,797 sets of data were collected from the database,

3. 41,728 predictions were made, where

4. 104 predictions exceeded the threshold.

Of the over forty thousand predictions, the median [IQR] time difference between admission and risk assessment was $1.3[0.9,32]$ minutes where $68 \%$ of predictions are made within five minutes of admission (database downtime causes delay where $<8 \%$ exceed six hours). Of 104 high-risk predictions, 27 were ill-timed (11 hospice admissions, 5 post-transplant admissions, and 11 encounters erroneously labeled 'inpatient'). From the 77 well-timed predictions, $50(65 \%)$ led to death or hospice within 60 days (median [IQR]: 25 [13, 61] days). Only 10 admissions (13\%) have no known end-of-life outcome at last censor (median [IQR]: 250 [60, 292] days). Live application of our model has identified patients at very high risk of short-term death within minutes of admission.

\section{Discussion \\ Prospective results and application feasibility}

Prospective implementation of the final model produces a total of 41,728 predictions over nine months. The $75 \%$ PPV operating threshold identifies 104 admissions $(0.25 \%)$ at very high risk. Although this proportion is small, it is consistent with other work and underscores how difficult it is to predict end-of-life with high confidence. The model is not perfect but neither is the standard of care. Many identified patients die within 60 days (65\%) and may have benefited from earlier, more comprehensive discussions about their goals of care. Future work will assess the ability of physicians to recognize which identified patients will not die and the impact of predictions upon clinical intervention. The model and prediction system are working as designed and will be expanded into practice to recommend supportive care.

\section{Generalization to Brooklyn cohort}

An evolving patient population is common in many applications and creates a practical challenge for prospective validation. In this case the mechanism of change is apparent: a new hospital was brought into the system that treats a new population with varying comorbidities and social determinants of health. Although this cause is obvious, the consequences are not. Along with an increase in proportion of patients observed at the Brooklyn hospital (38.0\% in testing up from 9.5\%; Table 1), there are corresponding structural differences in age, race, sex, outcome, and comorbidities between Manhattan and Brooklyn sites (eTable 1). These differences, indicative of a larger disparity between sites, further complicate generalization. Despite this, a model trained with only $10 \%$ of cases being from a new hospital can adapt to be performant and safely applied in a shifted patient mix.

The underrepresentation of Brooklyn patients during training does affect model performance at the Brooklyn hospital and their representation in the identified highrisk group (eFigures 5 and 6). A larger sample of Brooklyn patients for model training may improve the model's ability to learn the new site and improve performance. Any potential risk to patient safety is mitigated as only patient-positive interventions [25] will be applied to identified patients with no change in care for unidentified patients. However, it is unfair to Brooklyn patients to concentrate the intervention and its benefits to Manhattan. One recent model fairness work [22] has suggested the use of multiple thresholds, one for each sensitive group. This concept resembles affirmative action, especially when intentionally used to help overcome a well-established social challenge discernible 
within data. A Brooklyn-specific threshold would 'lower the bar' for Brooklyn patients to the same predefined $75 \%$ PPV (or lower) in an attempt to encourage adoption and more widespread use of supportive care in that community. A 75\% PPV threshold specific to Brooklyn was estimated at 0.295 which, when applied during the nine months of prospective validation, would have identified $450 \%$ more Brooklyn patients (55 vs. 10 ).

\section{Limitations}

Not all aspects of generalizability or model fairness could be assessed in this work. Some aspects that need further assessment include: 1) data collection that may be different between locations, 2) generalization to similar patients in different geographic locations, 3) application of the model to sites that use different EHR technologies, 4) more formal statistical methods to model multiple sites.

\section{Conclusion}

A machine learning model was developed and validated on retrospective patient data from three hospital sites. Assessment of model performance across sites and potentially sensitive demographics suggest varying degrees of unfairness as the one model and one operating threshold imperfectly learn differences in site, sex, race and ethnicity. Any degree of unfairness is ameliorated since the shift in performance at the underrepresented site raises the precision and sustains patient safety in the case of a patient-positive intervention. The model was implemented after initial testing reported the majority of cases were appropriate for intervention. When live, the model can deliver predictions within minutes of admission to prompt consideration by the care team and influence decision-making.

\section{Supplementary information}

Supplementary information accompanies this paper at https://doi.org/10. 1186/s12911-020-01235-6.

Additional file 1.

\section{Abbreviations}

AUPRC: Area under the precision-recall curve; AUROC: Area under the receiver operating characteristic; EHR: Electronic health record; IQR: Interquartile range; PPV: Positive predictive value; PRC: Precision-recall curve; ROC: Receiver operating characteristic

\section{Acknowledgements}

Not applicable.

\section{Authors' contributions}

VJM and YA formulated the study, processed results, wrote and revised the manuscript. VJM wrote code, analyzed results, prepared figures and tables, and implemented the live model. All authors read and approved the final manuscript.
Funding

None

\section{Availability of data and materials}

The underlying protected health information is not available. High-level data, such as that described in the figures and tables herein, are available upon reasonable request only if sharing that data would not endanger anyone's privacy.

\section{Ethics approval and consent to participate}

This project met the NYU Grossman School of Medicine Institutional Review Board (IRB) definition of quality improvement and therefore did not require IRB approval.

\section{Consent for publication}

Not applicable.

\section{Competing interests}

The authors declare that they have no competing interests.

Received: 12 May 2020 Accepted: 26 August 2020

Published online: 07 September 2020

\section{References}

1. Hui D, Kim SH, Roquemore J, Dev R, Chisholm G, Bruera E. Impact of timing and setting of palliative care referral on quality of end-of-life care in cancer patients. Cancer. 2014;120(11):1743-9 Available from: https://www.ncbi.nlm. nih.gov/pubmed/24967463.

2. Qaseem A, Snow V, Shekelle P, Casey DE Jr, Cross JT Jr, Owens DK, et al. Evidence-based interventions to improve the palliative care of pain, dyspnea, and depression at the end of life: a clinical practice guideline from the American College of Physicians. Ann Intern Med. 2008;148(2):141-6 Available from: https://www.ncbi.nlm.nih.gov/pubmed/18195338.

3. Rajaram A, Morey T, Dosani N, Pou-Prom C, Mamdani M. Palliative Care in the Twenty-First Century: using advanced analytics to uncloak insights from big data. J Palliat Med. 2019;22(2):124-5. https://doi.org/ 10.1089/jpm.2018.0609.

4. Christakis NA, Lamont EB. Extent and determinants of error in doctors' prognoses in terminally ill patients: prospective cohort study. BMJ. 2000; 320(7233):469-73 Available from: https://www.bmj.com/content/320/ 7233/469.short?casa_token=AxUA9Ee5DUsAAAAA:A6ttmBWRFgeMMjD3 GZ8ZW62j2sxWReeCBd_5r_zBN0LZUaLumgYQ8ytBY8LcpT0po_prbkWueBU5.

5. Glare $\mathrm{P}$, Virik $\mathrm{K}$, Jones $\mathrm{M}$, Hudson $\mathrm{M}$, Eychmuller $\mathrm{S}$, Simes J, et al. A systematic review of physicians' survival predictions in terminally ill cancer patients. BMJ. 2003;327(7408):195-8. https://doi.org/10.1136/bmj. 327.7408.195.

6. Amano K, Maeda I, Shimoyama S, Shinjo T, Shirayama H, Yamada T, et al. The Accuracy of Physicians' Clinical Predictions of Survival in Patients With Advanced Cancer. J Pain Symptom Manage. 2015;50(2):139-46.e1. https:// doi.org/10.1016/j.jpainsymman.2015.03.004.

7. Avati A, Jung K, Harman S, Downing L, Ng A, Shah NH. Improving palliative care with deep learning [Internet]. Vol. 18, BMC Medical Informatics and Decision Making. 2018. Available from: https://doi.org/ 10.1186/s12911-018-0677-8.

8. Wang L, Sha L, Lakin JR, Bynum J, Bates DW, Hong P, et al. Development and Validation of a Deep Learning Algorithm for Mortality Prediction in Selecting Patients With Dementia for Earlier Palliative Care Interventions [Internet]. JAMA Network Open. 2019;2:e196972. https://doi.org/10.1001/ jamanetworkopen.2019.6972.

9. Wegier P, Koo E, Ansari S, Kobewka D, O'Connor E, Wu P, et al. mHOMR: a feasibility study of an automated system for identifying inpatients having an elevated risk of 1-year mortality. BMJ Qual Saf. 2019. https://doi.org/10.1136/ bmjqs-2018-009285.

10. Makar M, Ghassemi M, Cutler DM, Obermeyer Z. Short-term mortality prediction for elderly patients using Medicare claims data. Int J Mach Learn Comput. 2015;5(3):192-7. https://doi.org/10.7763/IJMLC.2015.V5.506.

11. Elfiky A, Pany M, Parikh $R$, Obermeyer Z. A machine learning approach to predicting short-term mortality risk in patients starting chemotherapy [Internet]. bioRxiv. 2017:204081 Available from: https://www.biorxiv.org/ content/early/2017/10/17/204081.abstract. [cited 2017 Oct 25]. 
12. Brajer N, Cozzi B, Gao M, Nichols M, Revoir M, Balu S, et al. Prospective and External Evaluation of a Machine Learning Model to Predict In-Hospital Mortality of Adults at Time of Admission. JAMA Netw Open. 2020;3(2): e1920733. https://doi.org/10.1001/jamanetworkopen.2019.20733.

13. Curtis MD, Griffith SD, Tucker M, Taylor MD, Capra WB, Carrigan G, et al. Development and validation of a high-quality composite real-world mortality endpoint. Health Serv Res. 2018;53(6):4460-76 Available from: https://onlinelibrary.wiley.com/doi/abs/10.1111/1475-6773.12872.

14. Major VJ, Jethani N, Aphinyanaphongs Y. Estimating real-world performance of a predictive model: a case-study in predicting mortality. JAMIA Open. 2020 Apr 26 [cited 2020 Apr 27]; Available from: https://academic.oup.com/ jamiaopen/advance-article/doi/10.1093/jamiaopen/ooaa008/5825387.

15. Friedman J, Hastie T, Tibshirani R. Regularization paths for generalized linear models via coordinate descent. J Stat Softw. 2010;33(1):1 Available from: https://www.ncbi.nlm.nih.gov/pmc/articles/pmc2929880/

16. Chen T, Guestrin C. XGBoost: a scalable tree boosting system. In: proceedings of the 22nd ACM SIGKDD international conference on knowledge discovery and data mining [internet]. New York, NY, USA: Association for Computing Machinery; 2016 [cited 2020 Aug 13]. p. 785-94. (KDD '16). Available from: https://doi.org/10.1145/2939672.2939785.

17. Karampatziakis N. FEST: fast ensembles of sparse trees [Internet]. 2009. Available from: https://lowrank.net/nikos/fest/. Accessed 5 May 2017.

18. Olson RS, La Cava W, Mustahsan Z, Varik A, Moore JH. Data-driven advice for applying machine learning to bioinformatics problems [Internet]. arXiv [qbio.QM]. 2017. Available from: http://arxiv.org/abs/1708.05070. Accessed 30 Oct 2017.

19. Parikh RB, Manz C, Chivers C, Regli SH, Braun J, Draugelis ME, et al. Machine Learning Approaches to Predict 6-Month Mortality Among Patients With Cancer. JAMA Netw Open. 2019;2(10):e1915997. https://doi.org/10.1001/ jamanetworkopen.2019.15997.

20. Neto EC, Pratap A, Perumal TM, Tummalacherla M, Snyder P, Bot BM, et al. Detecting the impact of subject characteristics on machine learning-based diagnostic applications. npj Digital Medicine. 2019;2(1):1-6. Available from: https://www.nature.com/articles/s41746-019-0178-x.

21. Mitchell M, Wu S, Zaldivar A, Barnes P, Vasserman L, Hutchinson B, et al. Model cards for model reporting. In: Proceedings of the conference on fairness, accountability, and transparency [Internet]. 2019. p. 220-9. Available from: https://dl.acm.org/doi/abs/10.1145/3287560.3287596. Accessed 1 Feb 2020.

22. Corbett-Davies $\mathrm{S}$, Goel $\mathrm{S}$. The measure and mismeasure of fairness: a critical review of fair machine learning [Internet]. arXiv [cs.CY]. 2018. Available from: http://arxiv.org/abs/1808.00023. Accessed 15 Jan 2020.

23. Obermeyer Z, Powers B, Vogeli C, Mullainathan S. Dissecting racial bias in an algorithm used to manage the health of populations. Science. 2019; 366(6464):447-53. https://doi.org/10.1126/science.aax2342.

24. Gasparini A. comorbidity: An R package for computing comorbidity scores. J Open Source Software. 2018;3(23):648 Available from: https://joss.theoj.org/ papers/10.21105/joss.00648.pdf

25. Murray SG, Wachter RM, Cucina RJ. Discrimination By Artificial Intelligence In A Commercial Electronic Health Record-A Case Study. Health Affairs Blog. 2020. https://doi.org/10.1377/hblog20200128.626576.

\section{Publisher's Note}

Springer Nature remains neutral with regard to jurisdictional claims in published maps and institutional affiliations.

Ready to submit your research? Choose BMC and benefit from:

- fast, convenient online submission

- thorough peer review by experienced researchers in your field

- rapid publication on acceptance

- support for research data, including large and complex data types

- gold Open Access which fosters wider collaboration and increased citations

- maximum visibility for your research: over $100 \mathrm{M}$ website views per year

At BMC, research is always in progress.

Learn more biomedcentral.com/submissions 